\title{
Electronic transport properties and thickness dependence of the giant magnetoresistance in $\mathrm{Co} / \mathrm{Cu}$ multilayers
}

\author{
Th. Eckl, G. Reiss, a) H. Brückl, and H. Hoffmann \\ Institut für Angewandte Physik III, Universität Regensburg, Universitätsstrasse 31, 93053 Regensburg, \\ Germany
}

(Received 25 March 1993; accepted for publication 15 September 1993)

\begin{abstract}
The transport properties of electrons in $\mathrm{Co} / \mathrm{Cu}$ multilayered thin films are of special interest for the giant magnetoresistance (GMR) of this system. The magnitude of this effect depends on the mean free paths and on the strength of the interface scattering which in turn are strongly related to film structure. In this article, we discuss the results of resistance and magnetoresistance measurements carried out during film growth. We characterize the electronic transport parameters of these films and the growth mechanism of the layers. The new technique of the in situ measurement of the magnetoresistance furthermore provides a tool to find correlations of the growth mechanism with the dependence of the GMR on the Co thickness.
\end{abstract}

\section{l. INTRODUCTION}

During the last few years multilayered metallic structures have been under intensive investigation. One reason for these activities is the giant magnetoresistance (GMR) observed in multilayers consisting of ferromagnetic layers separated by nonmagnetic metal interlayers. ${ }^{1,2}$ For specific interlayer thicknesses (typically $10-20 \AA$ ) the magnetizations in successive ferromagnetic layers are antiparallel. ${ }^{2-5}$ This arrangement corresponds to a state of increased resistance. The reason for this correlation and therefore the GMR is under discussion.

Most of the theoretical work was applied to $\mathrm{Fe} / \mathrm{Cr}$ multilayered films, since the GMR was originally discovered in this system. ${ }^{1}$ Camley and Barnas ${ }^{6}$ used a semiclassical approach to describe the additional electron scattering in GMR structures. They calculated the resistivity by solving the Boltzmann transport equation. Bulk and interface scattering of the conduction electrons are expressed phenomenologically by introducing spin dependent mean free paths and boundary conditions with spin dependent transmission coefficients. This model is also used by other authors ${ }^{7,8}$ to describe the GMR in other systems. A quantum mechanical model with spin dependent interface- and bulk-scattering potentials has been discussed by Levy et al. ${ }^{9}$

To compare the numerical results from these theories with experimental data some specific parameters are required: the intrinsic mean free paths (IMFP) of the conduction electrons, the conductivities for thin layers of the materials, and the strength of the boundary scattering, which was first introduced by Fuchs ${ }^{10}$ using a specularity parameter $(p)$.

Since the bulk scattering as well as the interface scattering can be spin-dependent and therefore could contribute to the GMR, experimental investigations on the transport properties are required. The increase of the resistance for nonparallel layer magnetization in comparison with

\footnotetext{
${ }^{\text {a) }}$ Present address: IFF Dresden, Helmholtz Strasse 20, 01069 Dresden, Germany.
}

parallel magnetization should be apparent during growth of the multilayers. In situ resistance measurements, therefore, promise new experimental information on the origin of the GMR. This was demonstrated in a first attempt by composing in situ resistance versus thickness curves for different $\mathrm{Fe} / \mathrm{Cr}$ structures. ${ }^{\text {II }}$ In order to expand the possibilities of this approach, we additionally developed an in situ magnetoresistance measurement.

\section{EXPERIMENT}

The samples discussed in this article were prepared in ultrahigh vacuum (UHV) (base pressure $5 \times 10^{-9}$ ) by alternating thermal evaporation of $\mathrm{Co}$ and $\mathrm{Cu}$ on fire polished Corning ${ }^{\mathrm{TM}}$ glass at a deposition rate of $0.1 \AA \mathrm{A} / \mathrm{s}$. The substrate temperature was controlled at $300 \mathrm{~K}$ during evaporation. The film thickness was controlled by a quartz oscillator; the film resistance was measured with a pseudofour point method during evaporation.

We additionally employed a new technique of in situ magnetoresistance measurement where a variable computer-controlled magnetic field is applied to the sample during evaporation. In combination with the in situ measurement of the resistance, we were able to measure the dependence of the resistance on the magnetic field during evaporation within an increase in film thickness of less than $1 \AA$ during one cycle of the magnetic field.

The surface topography and the crystal structure of selected samples were examined by scanning tunneling microscopy (STM) and transmission electron microscopy (TEM). The STM images indicate a decreasing mesoscopic surface roughness with increasing number of layers and typical lateral crystallite sizes of $300 \AA$. The TEM pictures of sample cross sections show a well defined layered structure. The samples are polycrystalline with crystallites grown vertically through many layers. This suggests that both $\mathrm{Co}$ and $\mathrm{Cu}$ grew in the fec structure since this structure gives the smallest misfit of the lattices. 


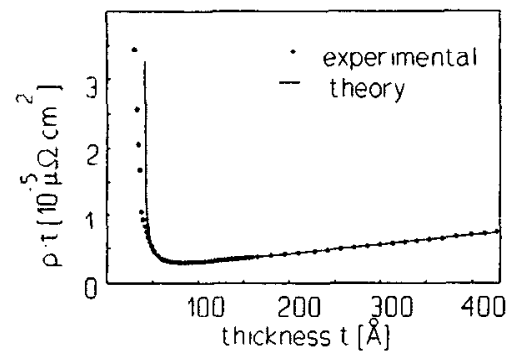

FIG. 1. $\rho(t) \times t$ vs $t$ curve of a single Co layer on glass (circles). The solid line is a fit of the Fuchs-Namba theory.

\section{RESULTS AND DISCUSSION}

\section{A. Transport parameters}

Transport parameters were determined from fitting the Fuchs-Namba theory ${ }^{10,12}$ to measured resistivity versus thickness data for single $\mathrm{Co}$ and $\mathrm{Cu}$ films. The fitting of the theoretical curves to the experimental data was done by a variation of the four parameters $l_{\infty}$ (IMFP), $\rho_{\infty}$ (bulk resistivity), $p$ (specularity parameter), and $H$ (amplitude of the mesoscopic surface roughness) ${ }^{13-15}$ Figure 1 shows a plot of the product $\rho \times t$ (resistivity times film thickness) versus the thickness $t$ of a single Co film. The values for the transport parameters received from fits to a large number of in situ measured resistivity versus thickness curves are given in Table I. Although the mesoscopic surface roughness $H$ of a single layer differs from the surface and interface roughnesses of multilayers (the roughnesses of $\mathrm{Co}$ and $\mathrm{Cu}$ on glass are distinctly larger than those of layers grown on metallic base layers which indicates that in this case the growth modes of $\mathrm{Co}$ and $\mathrm{Cu}$ are mostly a result of the substrate material and not of the substrate temperature), the values for $l_{\infty}$ and $\rho_{\infty}$ are independent of the roughness. The IMFPs of $\mathrm{Co}$ and $\mathrm{Cu}$ thin films are distinctly larger than the typical single layer thicknesses $t$ in $\mathrm{Co} / \mathrm{Cu}$ GMR multilayers, ${ }^{2}$ suggesting that conduction electrons can travel from one interface of a layer to the other without being scattered within this layer. This fact is important for the interpretation of the GMR as spin dependent scattering of the conduction electrons.

\section{B. Covering experiments}

From in situ resistance and simultaneous thickness measurements during the deposition of single films and multilayers we obtained information about the growth mechanisms of $\mathrm{Co}$ growing on $\mathrm{Cu}$ and $\mathrm{Cu}$ growing on $\mathrm{Co}$.

TABLE I. Transport parameters of single $\mathrm{Co}$ and $\mathrm{Cu}$ layers on glass. $(T=300 \mathrm{~K}$, deposition rate $0.1 \AA / \mathrm{s})$.

\begin{tabular}{lcc}
\hline \hline & $\mathrm{Co}$ & $\mathrm{Cu}$ \\
\hline$\rho_{\star}\left(10^{-6} \Omega \mathrm{cm}\right)$ & $14.0 \pm 0.5$ & $4.1 \pm 0.3$ \\
$1_{\star}(\mathrm{nm})$ & $22 \pm 2$ & $24 \pm 2$ \\
$p$ & $0.06 \pm 0.05$ & $0.6 \pm 0.05$ \\
$H(\mathrm{~nm})$ & $4 \pm 1$ & $6 \pm 1$ \\
\hline
\end{tabular}

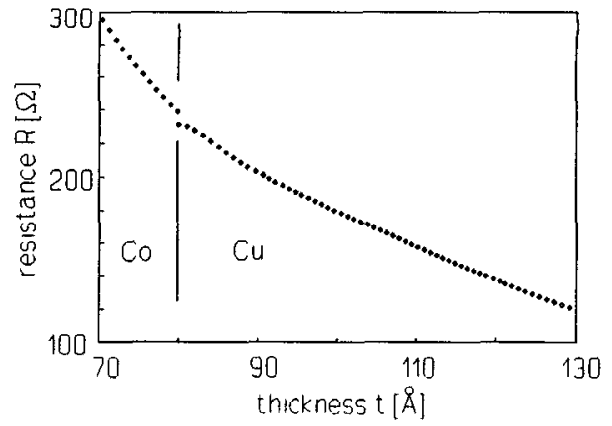

FIG. 2. Section of an in situ measured resistance vs thickness curve for a $\mathrm{Co}_{80} \AA / \mathrm{Cu}_{57 \AA} / \mathrm{Co}_{40 \AA} \AA$ film.

\section{1. $\mathrm{Cu}$ on $\mathrm{Co}$}

Since almost all conduction electrons approaching the Co surface are scattered diffusely (see Table I), ${ }^{12}$ adsorbed $\mathrm{Cu}$ atoms cannot increase the surface scattering. The resistance of a Co film should therefore not increase if it is covered with a small amount of $\mathrm{Cu}$ adatoms. Figure 2 shows a section of an in situ measured resistance versus thickness curve of a $\mathrm{Co}_{80 \AA} / \mathrm{Cu}_{57} \AA$ structure. The discontinuity in the resistance at the transition from $\mathrm{Co}$ to $\mathrm{Cu}$ results from relaxation processes during the time needed to stop the deposition of one material and to restart the deposition of the other. In agreement with the results obtained for single films, the resistance of the Co film does not show an increase during the covering with $\mathrm{Cu}$. The decrease of the resistance during $\mathrm{Cu}$ deposition can therefore be solely ascribed to the $\mathrm{Cu}$ layer. Therefore further evaluation is possible: the clear decrease in resistance within the first $5 \AA$ of $\mathrm{Cu}$ indicates that the $\mathrm{Cu}$ atoms are distributed homogeneously over the Co base layer. The application of a model of parallel resistors

$$
\frac{1}{R_{\mathrm{Cu}}}=\frac{1}{R}-\frac{1}{R_{b}}
$$

allows us to obtain the thickness-dependent resistivity of the Cu layer $\rho_{\mathrm{Cu}}\left(t_{\mathrm{Cu}}\right)\left(R_{\mathrm{Cu}}\right.$ : resistance of the Cu layer, $R_{b}$ : resistance of the base layers, $R$ : resistance of all layers). Figure 3 shows a typical $\rho_{\mathrm{Cu}}$ vs $t_{\mathrm{Cu}}$ curve of a Cu layer on a $\mathrm{Co}_{80} \AA$ film. The plateau in this curve between 8 and 20 $\AA$ layer thickness indicates that the $\mathrm{Cu}$ layer grows in an island-like mode within this thickness range. Above $20 \AA$ the resistivity decreases as the islands coalesce. STM investigations imply that the $\mathrm{Cu}$ smoothes the original surface roughness of the underlying Co layer, which according to our fitting results and STM investigations, has a surface roughness of about $40 \AA$. We suggest a picture where the $\mathrm{Cu}$ islands grow preferentially at the grain boundaries of the underlying layer (Fig. 4). This smoothing effect of the $\mathrm{Cu}$ layers is also confirmed by investigations of multilayers. Figure 5 shows a $\rho$ vs $t$ plot for successive Cu layers of a $\left[\mathrm{Co}_{80} \AA / \mathrm{Cu}_{57 \AA} \mathrm{A} /\left(\mathrm{Co}_{40} \AA / \mathrm{Cu}_{57} \AA\right) \times 6\right]$ multilayer, where the resistivity is evaluated with the model of parallel resistors. Obviously the plateau occurring for the first $\mathrm{Cu}$ layer vanishes with increasing number of layers. This indicates 


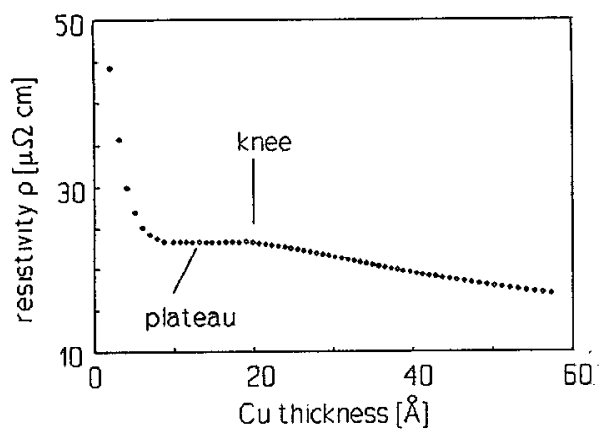

FIG. 3. Resistivity vs thickness curve of a Cu layer on a $80-\AA$-thick Co base layer. The resistance of the Co layer is eliminated with a model of parallel resistors.

that the island-like growth of the $\mathrm{Cu}$ layers is reduced, because the underlying surface becomes smoother.

These results strongly indicate a Stranski-Krastanovlike growth mechanism with small $\mathrm{Cu}$ islands growing mainly at the grain boundaries of the underlying Co layer, smoothing the mesoscopic surface roughness with increasing number of layers.

\section{Co on $\mathrm{Co} / \mathrm{Cu}$}

Figure 6 shows a typical $R$ vs $t$ curve measured during the deposition of $\mathrm{Co}$ atoms on a $\mathrm{Cu}$ layer. An obvious difference to the covering behavior of $\mathrm{Co}$ with $\mathrm{Cu}$ is the clear increase in the resistance when the first few $\AA$ 's of Co are deposited on the $\mathrm{Cu}$ base layer. Since the specularity parameter $p$ of a $\mathrm{Cu}$ layer is relatively large $(p=0.6$, see Table I), the Co adatoms can act as scattering centers and increase the diffuse surface scattering rate of the conduction electrons at the Cu surfacc. Because the maximum of the resistance is typically found at a Co thickness of $1.7 \AA$, independent of the thickness of the underlying $\mathrm{Cu}$ layer, the Co adatoms seem to build a very thin continuous layer on the $\mathrm{Cu}$.

The course of the $R$ vs $t$ curves is qualitativly independent of the measured magnetoresistance. This seems reasonable for our films as the MR ratios for sandwich structures do not exceed 4\% (see Sec. IV).

To determine the dependence of the height of the maximum of the resistance on the thickness of the underlying $\mathrm{Cu}$ layer, the resistance of the $\mathrm{Co}$ base layer was eliminated with the model of parallel resistors. A $\mathrm{Cu}$ layer covered with $1.7 \AA$ Co can be described as a Cu layer with strong surface scattering. Therefore it is possible to compare the experimental results with the Fuchs-Sondheimer theory ${ }^{16}$

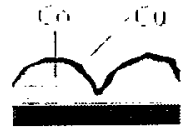

$t_{10}=4 \hat{A}$

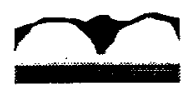

$\mathrm{t}_{\mathrm{Cu}}<20 \AA$

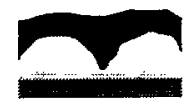

$\mathrm{t}_{\mathrm{Cu}}>20 \AA$
FIG. 4. Sketch of the suggested growth mechanism of $\mathrm{Cu}$ on $\mathrm{Co}$.

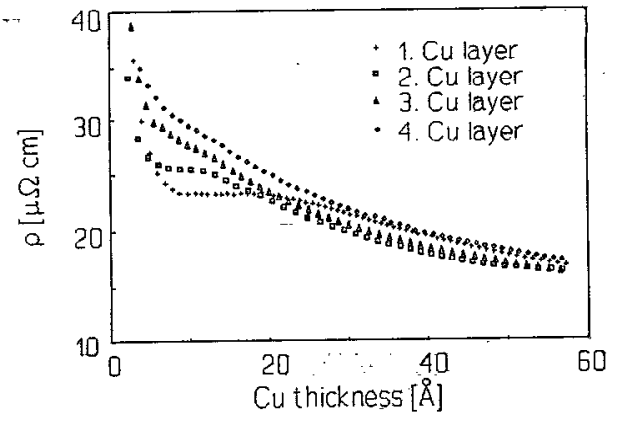

FIG. 5. Resistivity vs thickness curves for different $\mathrm{Cu}$ layers of a $\mathrm{Co} / \mathrm{Cu}$ multilayer. The resistance of the base layers is eliminaled with a model of parallel resistors.

which gives the following expression for the difference $\Delta R$ between the resistance of the uncovered $\mathrm{Cu}$ layer (length 1 , width $b$, thickness $t$ ) and the resistance of the $\mathrm{Cu}$ layer covered with Co:

$$
\Delta R=R_{\mathrm{cov}}-R_{\mathrm{uncov}}=\frac{3}{8} \frac{l}{b} \frac{l_{\infty} \rho_{\infty}}{t^{2}} \Delta p
$$

with

$$
\Delta p=p_{\text {uncov }}-p_{\text {cov }} .
$$

Figure 7 shows the experimental results and theoretical curves for different values of $\Delta p$. For $t_{\mathrm{Cu}}>30 \AA$ there is good agreement for $\Delta p \simeq 0.35$, whereas $\Delta R$ for thin $\mathrm{Cu}$ layers differs from the theory. This again points to a mesoscopic surface roughness of the underlying Co layer (see above) which prevents the formation of a closed homogeneous $\mathrm{Cu}$ layer thinner than $20 \AA$. This roughness cannot be described with the Fuchs-Sondheimer approximation. At about 3-4 $\AA$ Co thickness there is a turning point in the resistivity versus thickness curve (Fig. 6 ) indicating the completion of a first homogeneous Co layer. If the Co grew in monolayers, we would expect oscillations in the resistance curve with the period of the Co crystal structure, as the surface scattering and therefore the resistance would be reduced when an atomic layer is completed. ${ }^{15}$ We did not observe, however, such oscillations. On the other hand, the $\rho_{\mathrm{Co}} \mathrm{vs} t_{\mathrm{Co}}$ curves give no hint of island growth for larger Co thicknesses like the curves obtained for $\mathrm{Cu}$ on $\mathrm{Co}$. Al-

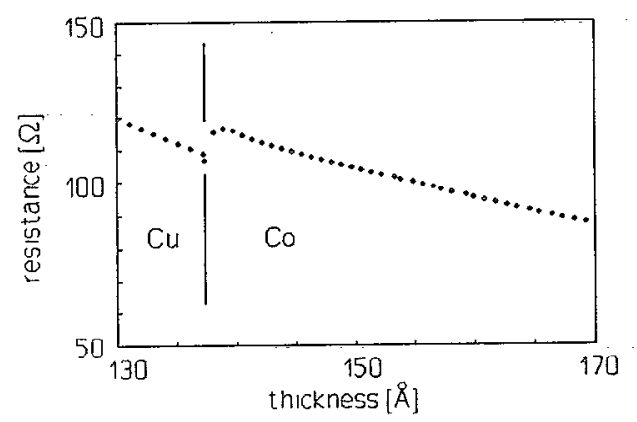

FIG. 6. Section of an in situ measured resistance vs thickness curve of a $\mathrm{Co}_{80 \AA} / \mathrm{Cu}_{57 \AA} / \mathrm{CO}_{40} \AA$-film. 


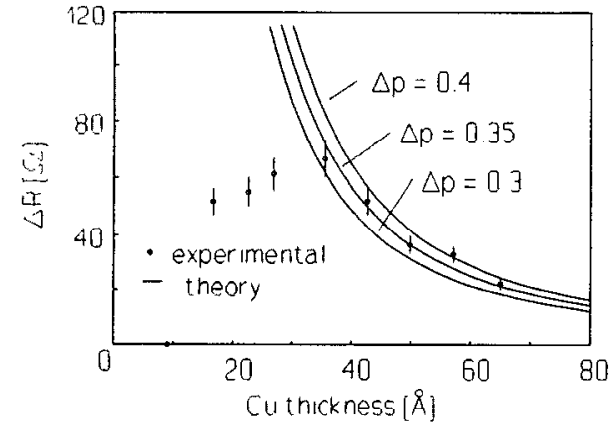

FIG. 7. Comparison of the $\mathrm{Cu}$ thickness dependent increase in resistance during the covering of $\mathrm{Cu}$ with $\mathrm{Co}$ and the theoretical results of the Sondheimer approximation. The $\mathrm{Cu}$ with the $\mathrm{Co}$ adatoms was treated as a $\mathrm{Cu}$ layer with increased surface scattering. The resistance of the base layers is eliminated in a model of parallel resistors.

though the $\mathrm{Co}$, therefore apparently does not show a real layer by layer growth on $\mathrm{Cu}$, there is no evidence of a substantial change in the surface profile due to the coverage of $\mathrm{Cu}$ with $\mathrm{Co}$.

In multilayers, the resistance versus thickness curves qualitatively show the same behavior except for the reduction of the surface roughnesses already discussed.

\section{Magnetoresistance measurements}

First we investigated the dependence of the GMR on the thickness of the intermediate $\mathrm{Cu}$ layer of $\mathrm{Co}_{80} \AA / \mathrm{Cu}_{x} / \mathrm{Co}_{40} \AA$ sandwich layers grown on glass substrates. The result is shown in Fig. 8. Distinct maxima of the magnetoresistance are found at $\mathrm{Cu}$ layer thicknesses of 43,53 , and $63 \AA$, respectively, giving an oscillation of the GMR with a period of about $10 \AA$. For multilayered films this oscillation was reported by other authors, $2,17,18$ who found periods of $10-12 \AA$ for $\mathrm{Co} / \mathrm{Cu}$ multilayers. A difference lies in the location of the maxima. For multilayers the first maximum was found at about $10 \AA \mathrm{Cu}$ thickness, whereas we found a much lower first maximum at $43 \AA$. This can be explained by the results presented above: since the first Co layer on the substrate shows a relatively large surface roughness, the $\mathrm{Cu}$ layer grown on this Co layer must be thicker in order to obtain a closed layer preventing

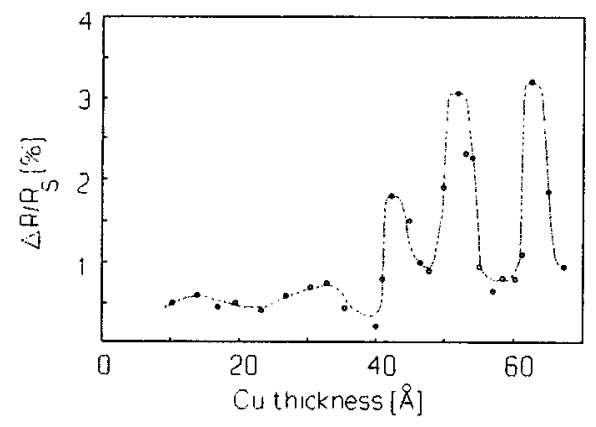

FIG. 8. Dependence of the MR on the thickness of the $\mathrm{Cu}$ interlayer in $\mathrm{Co}_{80} \mathrm{~A} / \mathrm{Cu}_{7} / \mathrm{Co}_{30}$-films. adjacent Co layers from direct exchange-coupling (unfortunately our vibrating sample magnetometer measurements gave no clear evidence for ferromagnetic or antiferromagnetic interlayer coupling). Thus the great surface roughness of the base layers hinders the development of GMR for $\mathrm{Cu}$ thickncsses smaller than $40 \AA$ in these trilayers. This seems to be contradictory to the results on the $\mathrm{Fe} / \mathrm{Cr}$ system where an increase in MR with increasing interface roughness has been reported. ${ }^{19}$ Unfortunately no values for the roughness amplitudes and the characteristic lateral distances have been reported, but we think that the interface roughness amplitudes (microscopic roughness) leading to an increase in MR must be distinctly smaller than the roughness amplitudes (mesoscopic roughness) treated in our work.

\section{In situ magnetoresistance measurements}

In order to obtain detailed information about the development of GMR during film growth, we developed an in situ magnetoresistance measurement (IMM) technique. An in plane magnetic field $H$ was applied to the sample and varied from -850 to 850 Oe within $1 \mathrm{~s}$. Since the saturation field (smaller than 250 Oe for $t>10 \AA$ ) of the investigated samples is smaller than the maximum of the applied field, we were able to obtain a complete $R$ vs $H$ curve during the deposition within a thickness increase of only $0.1 \AA$. To eliminate anisotropic MR effects, the magnetic field is directed parallel to the electric field in the film. From this we derived the dependence of the magnetoresistance ratio on the film thickness $t$ :

Magnetoresistance $(t)=\frac{\max \left[R(H, t)-R_{s}(t)\right]}{R_{s}(t)}=\frac{\Delta R(t)}{R_{s}(t)}$.

Figures $9(a)-9(\mathrm{c})$ show typical resistance versus thickness curves for different magnetic fields and the resulting IMM curve for the second Co layer of a $\mathrm{Co}_{80} \AA / \mathrm{Cu}_{50 \AA} / \mathrm{CO}_{780} \AA$-trilayer. For the first Co layer (not shown in Fig. 9) and the $\mathrm{Cu}$ interlayer the MR shows negative values smaller than $0.5 \%$, which can be attributed to the anisotropic MR.

When the deposition of the second Co layer is started, the MR becomes positive at about $1.6 \AA$ Co thickness, which coincides with the maximum in resistance (see above). This suggests that already the first atomic layer of Co has a clear influence on the magnetoresistive behavior of this films. At about 3-4 $\AA$ Co thickness there is a first maximum in the MR, which we observed in all films showing an increased MR. Following this first peak there is a steep increase in the MR, reaching a main maximum at about $13 \AA$ Co layer thickness. The position of this maximum lies between 11 and $15 \AA$ for different films and does not depend on the interlayer thickness. This indicates that the MR has fully evolved within the first $13 \AA$ Co thickness and that further increase in Co thickness decreases the MR by shunting the scattering effects in the underlying layers. 


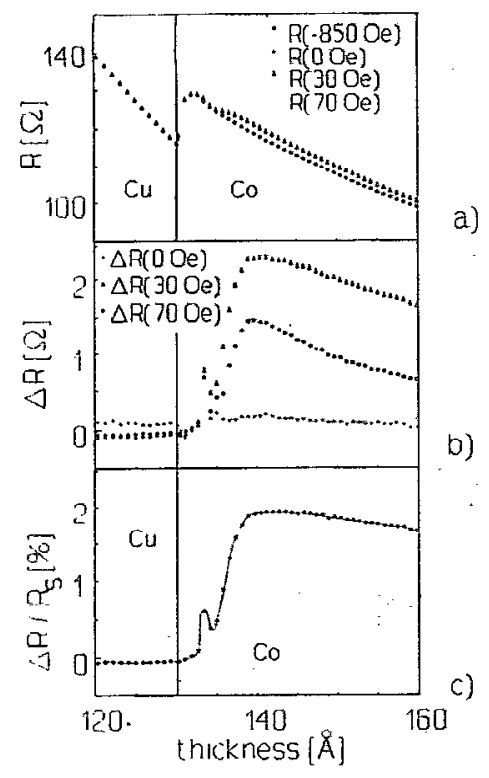

FIG. 9. (a) In situ measured resistance vs thickness curves of a $\mathrm{CO}_{80} \mathrm{~A} / \mathrm{Cu}_{50} \mathrm{~A} / \mathrm{CO}_{780 \AA}$ film for different magnetic fields. (b) Difference between the thickness-dependent resistances for different magnetic fields and the thickness-dependent resistance for a field bigger than the saturation field $\Delta R(H, t)=R(H, t)-R_{s}\left(H>H_{s}, t\right)$. (c) In situ magnetoresistance.

On the basis of the results obtained from trilayers we also applied IMM to multilayered films. We obtained a $\Delta R / R$ ratio of about $6 \%$ at room temperature for a $\left[\mathrm{CO}_{80 \AA} /\left(\mathrm{Cu}_{20} \AA / \mathrm{Co}_{15} \AA\right) \times 7\right]$ film, which is relatively low compared to values reported by other authors. ${ }^{2,17,20}$ Figure 10 shows the IMM result obtained for a $\left[\mathrm{CO}_{70} \AA /\left(\mathrm{Cu}_{20} \AA / \mathrm{Co}_{15} \AA\right) \times 7\right]$ multilayer. This measurement supports our thesis that the mesoscopic surface roughness of $\mathrm{Co}$ on glass prevents the evolution of an increased MR for $\mathrm{Cu}$ interlayers thinner than $40 \AA$. While the surface roughness decreases with increasing number of layers (see above), the GMR clearly increases. In the example shown in Fig. 10, the 6th Co layer gives rise to an increase in MR from $0.5 \%$ to $3 \%$. An evaluation of the MR of this trilayer (5th $\mathrm{Co} / \mathrm{Cu} / 6$ th $\mathrm{Co}$ layer) with the model of parallel resistors gives a GMR of $14 \%$, which is

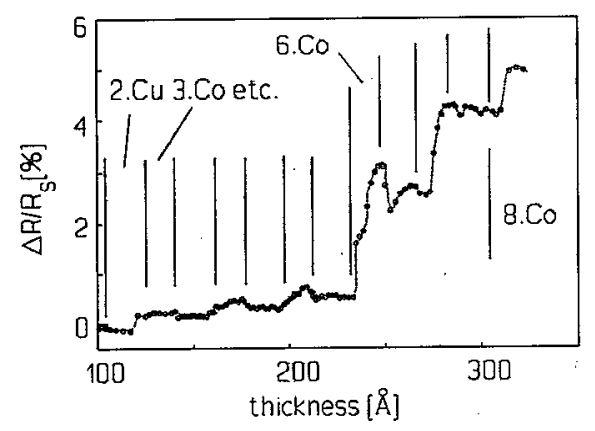

FIG. 10. In situ measured magnetoresistance vs thickness curve of a $\left[\mathrm{Co}_{70} \AA /\left(\mathrm{Cu}_{20} \AA / \mathrm{Co}_{15 \mathrm{~A}}\right) \times 7\right]$ multilayer. distinctly larger than the $3 \%$ obtained for trilayers grown on glass. Further IMM measurements on films grown on different substrates and in different geometries are in progress.

\section{CONCLUSIONS}

In summary, we carried out in situ resistance measurements in order to obtain information about the electronic transport parameters and the growth mode of polycrystalline $\mathrm{Co} / \mathrm{Cu}$ multilayered thin films on glass. We obtained strong evidence for Stransky-Krastanov-like growth of $\mathrm{Cu}$ on $\mathrm{Co}$, with preferred island nucleation at the grain boundary grooves of the Co film. In contrast, Co seems to reproduce the surface profile of an underlying $\mathrm{Cu}$ film. The transport of the conduction electrons in $\mathrm{Co} / \mathrm{Cu}$ multilayers is characterized by mean free paths of about $23 \mathrm{~nm}$ in both matcrials. The scattering of the conduction electrons at the free Co surfaces, however, is much stronger than at the free $\mathrm{Cu}$ surface. Therefore only the $\mathrm{Co}$ atoms on the $\mathrm{Cu}$ surfaces act as additional scattering centers for the conduction electrons. In trilayers, the GMR can be observed only for $\mathrm{Cu}$ thicknesses larger than about $4 \mathrm{~nm}$ due to the relatively large roughness of the first Co film on glass. As the number of layers is increased, the surface roughness decreases and in turn the GMR obtained for each individual $\mathrm{Co} / \mathrm{Cu} / \mathrm{Co}$ structure of the multilayer increases.

Furthermore we developed a new in situ magnetoresistance measurement (IMM) which provides more detailed information about the thickness dependent evolution of the MR. The MR reaches a distinct maximum at about $13 \AA$ Co thickness, indicating that the scattering processes causing the GMR take place within a very narrow region at the interfaces of the multilayered structure.

In general the result that a mesoscopic surface roughness can prevent the evolution of an increased MR seems contradictory to recent findings, ${ }^{19}$ indicating that a specific increase in surface roughness increases MR. But we think that the roughnesses of our Co base layers are too big to get thin continuous $\mathrm{Cu}$ interlayers which is necessary for an antiparallel allignment of adjacent Co layers. For thicker $\mathrm{Cu}$ interlayers $(t>30 \AA)$ and for multilayers the MR is decreased by shunting.

${ }^{1}$ M. N. Baibich, J. M. Broto, A. Fert, F. Nguyen Van Dau, and F. Petroff, Phys. Rev. Lett. 61, 2472 (1988).

${ }^{2}$ S. S. P. Parkin, R. Bhadra, and K. P. Roche, Phys. Rev. Lett. 6, 2152 (1991).

${ }^{3}$ S. Araki, K. Yasui, and Y. Narumiya, J. Phys. Soc. Jpn. 60, 2827 (1991).

${ }^{4}$ A. Cebollada, J. L. Martinez, J. M. Gallego, J. J. de Miguel, R. Miranda, S. Ferrer, F. Batallan, G. Fillion, and J. P. Rebouillat, Phys. Rev. B 39, 9726 (1989).

${ }^{5}$ P. Grünberg, R. Schreiber, Y. Pang, M. B. Brodsky, and H. Sowers, Phys. Rev. Lett. 57, 2442 (1986).

${ }^{6}$ R. E. Camley and J. Barnas, Phys. Rev. Lett. 63, 664 (1989).

${ }^{7}$ A. Barthelemy and A. Fert, Phys. Rev. B 43, 13124 (1991).

${ }^{8} \mathrm{~J}$. Inoue, A. Oguri, and S. Maekawa, J. Magn. Magn. Mater. 104-107, 1883 (1992).

${ }^{9}$ P. M. Levy, S. Zhang, and A. Fert, Phys. Rev. Lett. 65, 1643 (1990).

${ }^{10}$ K. Fuchs, Proc. Camb. Philos. Soc. 34, 100 (1938).

${ }^{11}$ M. Jacob, G. Reiss, H. Brückl, and H. Hoffmann, Phys. Rev. B 46, 11208 (1992). 
${ }^{12}$ Y. Namba, Jpa. J. Appl. Phys. 9, 1326 (1970).

${ }^{15} \mathrm{~J}$. Vancea, $G$. Reiss, and $H$. Hoffmann, Phys. Rev. B 35, 6435 (1987).

${ }^{14}$ G. Reiss, K. Kapfberger, G. Meier, J. Vancea, and H. Hoffmann, $J$. Phys. Condens. Matter 1, 1275 (1989).

15 U. Jacob, J. Vancea, and H. Hoffmann, Phys. Rey. B 41, 11852 (1990)

${ }^{16} \mathrm{E}$. H. Sondheimer, Adv. Phys. 1, ] (1952).
${ }^{17}$ D. H. Mosca, F. Petroff, A. Fert, P. A. Schroeder, W. P. Pratt, and R. Laloee, J. Magn. Magn. Mater. 94, L1 (1991).

${ }^{18}$ S. S. P. Parkin, Phys. Rev. Lett. 67, 3598 (1991).

${ }^{19}$ E. Fullerton, D. M. Kelly, J. Guimpel, I. K. Schuller, and Y. Bruynseraede, Phys. Rev. Lett. 68, 859 (1992).

${ }^{20}$ T. R. McGuire and T. S. Plaskett (preprint). 